\title{
Multimodal Imaging of the Distal Interphalangeal- joint Synovio-entheseal Complex in Psoriatic Arthritis (MIDAS): a Cross-sectional Study on the Diagnostic Accuracy of Different Imaging Modalities Comparing Psoriatic Arthritis to Psoriasis and Osteoarthritis
}

Jørgen Guldberg-Møller ( $\sim$ joergen.guldberg-moeller@regionh.dk )

Parker Instituttet https://orcid.org/0000-0002-6521-5825

Mette Mogensen

Bispebjerg Hospital

Karen Ellegaard

Parker Instituttet

Ali Zavareh

Guy's and Saint Thomas' Hospitals NHS Trust: Guy's and St Thomas' NHS Foundation Trust

Richard J. Wakefield

University of Leeds Leeds Institute of Biomedical Clinical Sciences

Ai Lyn Tan

University of Leeds Leeds Institute of Biomedical Clinical Sciences

Mikael Boesen

Bispebjerg Hospital

Jamshid Dehmeshki

Image Analysis Group

Olga Kubassova

Image Analysis Group

Lene Dreyer

Aalborg Hospital: Aalborg Universitetshospital

Marius Henriksen

Parker Instituttet

Lars Erik Kristensen

Parker Instituttet 
Keywords: Psoriatic arthritis, nail, enthesis, colour Doppler ultrasonography, magnetic resonance imaging, X-ray

Posted Date: May 6th, 2021

DOI: https://doi.org/10.21203/rs.3.rs-486995/v1

License: (1) This work is licensed under a Creative Commons Attribution 4.0 International License. Read Full License

Version of Record: A version of this preprint was published at RMD Open on March 1st, 2022. See the published version at https://doi.org/10.1136/rmdopen-2021-002109. 


\section{Abstract}

\section{Background}

No gold-standard diagnostic test for psoriatic arthritis (PsA) exists. The diagnosis relies on different patterns of symptom involvement which can mimic other arthritides such as hand osteoarthritis (OA). We aimed to investigate if ultrasound (US), magnetic resonance imaging (MRI) and X-Ray applied to the distal interphalangeal (DIP)-joint and synovio-entheseal complex (SEC) can be used to discriminate between patients with PsA, skin psoriasis (PsO) and OA.

\section{Methods}

In this prospective, cross-sectional study, patients with DIP-joint PsA and nail involvement ( $n=50)$, PsO with nail involvement $(n=12)$; and OA $(n=13)$; were consecutively recruited. Risk ratios (RR) and odds ratios were calculated for US, MRI and X-ray findings of the DIP-joint and SEC between diagnoses.

\section{Results}

New bone formation (NBF) assessed by US and MRI was a hallmark of OA, reducing the risk of having PsA (RR 0.52 (CI95\% 0.43 to 0.63 ) and 0.64 (CI95\% 0.56 to 0.74). The OA group was different from PsA and PsO on all MRI semiquantitative and X-ray outcomes reflected in a much lower RR of having PsA; RR ranging from 0.20 ( $\mathrm{Cl} 95 \% 0.13$ to 0.31 ) for MRI bone marrow oedema to 0.85 ( $\mathrm{Cl} 95 \% 0.80$ to 0.90 ) in Xray entheseal bone-change. No outcome in US, MRI or X-ray was associated with a higher risk of PsA versus $\mathrm{PsO}$, although the damage grade was higher in $\mathrm{PsA}$.

\section{Conclusions}

The differentiation between PsA, PsO, and OA using US, MRI and X-ray is possible based on the grade of structural disease involvement using semiquantitative OMERACT US scores, MRI PsAMRIS score and Xray score. A high grade of US, MRI and X-ray NBF and MRI bone marrow oedema reduce the RR of having PsA compared to OA. In demarcating PsA from PsO patients, it is of significance if they present with Xray enthesitis and a high degree of US erosions and NBF or MRI synovitis-, tenosynovitis-, erosion score or bone marrow oedema.

\section{Introduction}

Psoriatic arthritis (PsA) is seen in 5-40\% of patients with psoriasis (PsO) (1) with a typical delay of 7 years $(2,3)$, and the incidence has been rising in the last two decades (4). The transition from inflammatory skin disease to a heterogeneous and widespread joint disease (5-7) represents a shared diagnostic challenge in dermatology and rheumatology. As no gold-standard diagnostic test for PsA exists, establishing the diagnosis relies on different patterns of clinical, radiological and serological markers expressed in the CASPAR-criteria (8). Also, some typical features of PsA can mimic other arthritides such as rheumatoid arthritis (RA) (9) and hand osteoarthritis (OA), which can cause a 
significant delay of the diagnosis with a potential impact on therapy for patients and costs to society $(10,11)$. RA rarely affects the distal interphalangeal (DIP)-joint (12), but both bone proliferation and inflammation processes in the DIP-joint are shared entities in PSA and OA $(13,14)$.

Nail dystrophy is a prognostic marker of DIP-joint arthritis (15), with a prevalence between $41-93 \%$ in PsA and $15-50 \%$ in PsO (16-18). This suggests that nail dystrophy can serve as an early diagnostic entity to identify the transition from PsO to PsA. A proposed anatomical relationship between the nail and DIPjoint through the extensor tendon and joint capsule suggests that psoriatic nail manifestations are extensions of enthesopathy in the neighbouring structures, referred to as the synovio-entheseal complex $(\operatorname{SEC})(14,19,20)$.

Comparisons of different objective disease markers between PsO, PsA, and OA may allow an improved understanding of the transition from PsO to PsA and distinguish it from OA. A range of imaging modalities have been applied to explore the nail, DIP joints and SEC in PSA.

Magnetic Resonance Imaging (MRI) studies support the SEC-theory as DIP joint disease in PsA is associated with entheseal inflammation, suggesting that the entheses are the epicentre for inflammatory changes in PsA DIP-joint disease (14).

Ultrasonography (US) has demonstrated an association between extensor tendon enthesopathy and clinical nail involvement (21). Nail pitting and onycholysis in PsO have been associated with a higher risk of subsequent transition to DIP-joint arthritis and PsA $(15,22)$.

Although X-ray has been less sensitive than US and MRI in evaluating chronic changes in arthritis $(23,24)$, such as erosions and new bone formation (NBF), it is frequently used in the initial diagnostic workup in a patient suspected of inflammatory arthritis. Diagnostic patterns of destructive or prolific changes in X-ray thus play a vital role in the initial differentiation of arthritides.

While these different imaging modalities have been applied in separate studies to explore the nail and SEC, they have never been applied in combination in the same anatomical region in related disease entities. By doing so, distinct patterns of joint-, nailfold- and entheseal involvement may be revealed to more accurately distinguish subtle differences between PsA, PsO and Hand OA to diagnose PsA earlier.

The present study investigates if US, MRI, and X-Ray applied to the DIP-joint and SEC can be used to discriminate between patients with PsA, PsO and OA.

\section{Methods}

This prospective study included cross-sectional data on consecutively enrolled patients with PsA, PsO with nail involvement and hand OA. Only DIP-joints 2-5 of the dominant hand were assessed. The study followed the STARD-guidelines (25) and a prespecified protocol, see Supplementary Data S1. 
Between 1st of December 2017 and 1st of June 2020, patients with PsA according to the CASPAR criteria (8) and a Nail Psoriasis Severity Index (NAPSI) score $\geq 5$ was enrolled from three rheumatology outpatient clinics located in the Capital Region of Denmark and the Zealand Region in Denmark. Patients with $\mathrm{OA}$ and $\mathrm{PsO}$ were recruited from the Osteoarthritis-, rheumatology-and dermatology outpatient clinics at Bispebjerg and Frederiksberg Hospital. The PsO patients were diagnosed by a dermatologist and the OA patients by a rheumatologist according to the ACR criteria for hand OA (26).

For eligibility criteria, see Supplementary Table S2. Signed informed consent was obtained from all participants, and the study was approved by the local Ethics Committee of the Capital Region in Denmark on 25-05-2018, Journal-no.: H-18006696 and the Danish Data Protection Agency on 04-06-2018 (VD2018-149, med I-Suite no.: 6392).

\section{Patient-reported outcome measures (PROM)}

The physical function was addressed by the Health Assessment Questionnaire disability index (HAQ-DI) (27). The average joint-related pain, general fatigue and global assessment of disease impact within the last week were assessed by Visual Analog Scales 0-100 mm (VAS) with anchors: $0=$ no pain/fatigue/impact and $100=$ worst imaginable pain/fatigue/impact.

\section{Clinical examination}

A trained rheumatologist (JG) with nine years of clinical experience conducted: $66 / 68$ swollen/tender joint count, a Psoriatic Area Severity Index (PASI score), a full NAPSI score (score 0-8 on each nail), and Spondyloarthritis Research Consortium of Canada (SPARCC) Enthesitis Index (score 0-16, based on 9 bilateral sites; the inferior patella and tibial tuberosity are considered 1 site) (28). The clinician was blinded to other examinations.

\section{Ultrasound assessment}

US assessment was performed by an experienced (15 years) and certified sonographer (KE) blinded to the clinical results.

US assessments were performed in the longitudinal plane for DIP-joint 2-5 using a General Electric Logiq E9 (Milwaukee, Wisconsin, USA) and a linear array matrix transducer (9-15 MHz frequency). The choice of the probe was based on the consensus of international experts in ultrasonography in an unpublished web-survey (Supplementary Data S3).

The dorsal and volar aspects of $2^{\text {nd }}$ to $5^{\text {th }}$ DIP-joint was semi-quantitatively scored 0-3 for the presence of Grey-scale SH Color Doppler, NBF and erosions according to the OMERACT standards (29). Flexor- and extensor tendon, nail matrix, nail bed and nail thickness were measured in $\mathrm{mm}$. in the longitudinal plane. The ratio of colored/grey pixels in a predefined region of interest (ROI) was calculated (QAnalysis GE 
software ver. R6 2.0) and reported as Max-ratio and Min-ratio (30). All US images analysis was done blinded to diagnosis by JG. See Supplementary Data S4 for full US protocol.

\section{Magnetic resonance imaging}

The distal phalanges and DIP-joint from the $2^{\text {nd }}$ to $5^{\text {th }}$ fingers were examined in a 3 T Siemens Verio ${ }^{\circledR}$ MRI scanner using a semi-flex 16 channel body coil. A modified PsAMRIS-score was used in this study encompassing the DIP-joint: D-PsAMRIS range 0-35.

DCE-MRI images were analysed using DYNAMIKA® software (Image analysis group (IAG), London UK). A 3-dimensional ROI was drawn around the distal phalanges and DIP-joint from $2^{\text {nd }}$ to $5^{\text {th }}$ fingers. The computed output data in the various ROls comprise the mean of the initial rate of enhancement (IRE), maximum enhancement (ME), number of enhancing voxels (NVoxels) and composite scores: IRE*NVoxels and ME*Nvoxels.

The MRI analyses were performed by a radiologist (MB) with 15 years of experience blinded to diagnosis. For complete MRI protocol and DCE-MRI analysis method, see Supplementary Data S5.

\section{X-ray}

Plain posterior-anterior radiographs of the $2^{\text {nd }}$ to $5^{\text {th }}$ DIP-joint was evaluated for entheseal bone-change and erosions and scored 0-3 (0: no changes and 3: large changes). The images were evaluated by a trained radiologist (AZ) with five years of experience blinded to diagnosis. For protocol, see Supplementary Data S6.

\section{Statistics}

The number of PsA patients was predefined and based on power calculations in the protocol (Supplementary Data 1) to 50 participants, and OA and PsO to at least 10 and less than 20 subjects to detect changes that would be detectable but also occur at a meaningful clinical frequency.

The primary analyses focused on assessing differences between diagnoses (the reference standard) in US, MRI and X-ray assessments (index tests) of the SEC. We analysed all data, i.e. four fingers from each participant. The quantitative and semiquantitative US, MRI and X-ray outcomes were analysed using mixed linear models with diagnosis as a fixed factor (3 levels: PsA, PsO and OA) and participant as a random factor. The binominal outcomes were compared using Pearson Chi-square. Odds Ratio and Risk Ratio assessment were calculated using crosstabs, and differences odds ratios between groups were calculated by Mantel-Haenszel Common Odds Ratio Estimate. The diagnostic properties of the index tests: US, MRI and X-ray, was calculated using logistic regression analyses on the three groups pooled with diagnosis (PsA versus PsO and PsA versus OA) as a dependent variable and the quantitative US, 
MRI and X-ray outcomes as independent variables. The cut-off points to reliably diagnose PsA is determined using a receiver operating characteristic curve analysis estimating the area under the curve (AUC). No, imputation of missing data was performed.

Intra-observer reliability for the US scores was assessed on 100 randomly chosen DIP-joints scored twice by the same rater (JG) separated by 10 days. The intra-rater reliability was quantified using the intraclass correlation coefficient (ICC) $(31,32)$ and weighted Kappa. The intra-rater reliability was good (lowest ICC: 0.64 and lowest Kappa: 0.73; see Supplementary Table S7).

The analyses were conducted using IBM SPSS Statistics ver. 25.

\section{Results}

Out of 98 screened subjects, we included 50 patients with PsA, 12 with PsO and 13 with OA (Fig. 1). Demographics of the three groups are shown in Table 1. Patients with PsA and PsO were younger than $\mathrm{OA}$ patients and had a higher BMI. 
Table 1

Demographics and characteristics of PsA, PsO and OA patients. Data are presented as median [25th 75th percentiles] unless otherwise stated. All abbreviations are explained in the method section except

BMI: Body Mass Index, csDMARD: conventional Disease Modifying Anti-Rheumatic Drug, bDMARD: biological Disease Modifying Anti-Rheumatic Drug.

\begin{tabular}{|c|c|c|c|c|}
\hline & \multirow[t]{2}{*}{ Baseline variables } & \multirow{2}{*}{$\begin{array}{l}\text { PsA } \\
n=50 \\
(48 \%)\end{array}$} & \multirow{2}{*}{$\begin{array}{l}\text { PsO } \\
n=12(11 \\
\%)\end{array}$} & \multirow{2}{*}{$\begin{array}{l}\text { Hand OA } \\
n=13(12 \\
\%)\end{array}$} \\
\hline & & & & \\
\hline \multirow[t]{10}{*}{$\begin{array}{l}\text { Socio-Demography } \\
\text { /treatment }\end{array}$} & Females, n (\%) & $21(42 \%)$ & $5(41 \%)$ & $\begin{array}{l}13(100 \\
\%)\end{array}$ \\
\hline & Age, years, mean (SD) & $\begin{array}{l}54.4 \\
(12.0)\end{array}$ & $\begin{array}{l}55.3 \\
(18.0)\end{array}$ & $69.5(8.9)$ \\
\hline & Disease duration months & $\begin{array}{l}60 \\
{[19 ; 120]}\end{array}$ & $25[12 ; 96]$ & $78[12 ; 99]$ \\
\hline & Height, cm, mean (SD) & $\begin{array}{l}172.8 \\
(10.0)\end{array}$ & $\begin{array}{l}173.3 \\
(10.0)\end{array}$ & $\begin{array}{l}160.2 \\
(6.7)\end{array}$ \\
\hline & weight, kg, mean (SD) & $\begin{array}{l}85.7 \\
(16.8)\end{array}$ & $\begin{array}{l}82.6 \\
(16.3)\end{array}$ & $\begin{array}{l}62.7 \\
(10.7)\end{array}$ \\
\hline & $\mathrm{BMI} \mathrm{m} / \mathrm{kg}^{2}$, mean (SD) & $28.8(6.6)$ & $27.5(5.3)$ & $24.3(2.5)$ \\
\hline & Treated with csDMARDS, n (\%) & $30(60 \%)$ & $3(25 \%)$ & $1(7 \%)$ \\
\hline & Treated with bDMARDS, n (\%) & $4(8 \%)$ & $5(42 \%)$ & 0 \\
\hline & $\begin{array}{l}\text { Treated with both bDMARDS and } \\
\text { csDMARDS, } \mathrm{n}(\%)\end{array}$ & $7(14 \%)$ & 0 & 0 \\
\hline & No treatment, n (\%) & $9(18 \%)$ & $4(33 \%)$ & $12(93 \%)$ \\
\hline \multirow[t]{4}{*}{ PROM } & HAQ-DI (0-3) & $\begin{array}{l}0.44 \\
{[0.13 ; 0.88]}\end{array}$ & $\begin{array}{l}0.00 \\
{[0.00 ; 0.13]}\end{array}$ & $\begin{array}{l}0.63 \\
{[0.25 ; 0.88]}\end{array}$ \\
\hline & VAS Fatigue (0-100), mean (SD) & $\begin{array}{l}47.6 \\
(27.8)\end{array}$ & $\begin{array}{l}27.3 \\
(22.9)\end{array}$ & $\begin{array}{l}24.8 \\
(22.1)\end{array}$ \\
\hline & VAS Pain (0-100), mean (SD) & $\begin{array}{l}36.2 \\
(26.3)\end{array}$ & $9.5(10.8)$ & $\begin{array}{l}42.2 \\
(21.2)\end{array}$ \\
\hline & VAS Global (0-100), mean (SD) & $\begin{array}{l}44.5 \\
(28.0)\end{array}$ & $\begin{array}{l}14.6 \\
(17.5)\end{array}$ & $\begin{array}{l}40.7 \\
(22.9)\end{array}$ \\
\hline \multirow[t]{3}{*}{ Clinical measures } & Swollen joint count (0-66) & $\begin{array}{l}3.5 \\
{[1.0 ; 5.0]}\end{array}$ & $1.0[0 ; 3.0]$ & $\begin{array}{l}2.0 \\
{[1,0 ; 4.0]}\end{array}$ \\
\hline & Tender joint count $(0-68)$ & $\begin{array}{l}6.5 \\
{[1.0 ; 14.0]}\end{array}$ & $\begin{array}{l}0.0 \\
{[0.0 ; 2.0]}\end{array}$ & $\begin{array}{l}9.0 \\
{[5.0 ; 11.0]}\end{array}$ \\
\hline & PASI (0-72) & $\begin{array}{l}1.9 \\
{[0.8 ; 4.0]}\end{array}$ & $\begin{array}{l}1.8 \\
{[0.0 ; 6,1]}\end{array}$ & $\begin{array}{l}0.0 \\
{[0.0 ; 0.0]}\end{array}$ \\
\hline
\end{tabular}




\begin{tabular}{|c|c|c|c|}
\hline \multirow[t]{2}{*}{ Baseline variables } & \multirow{2}{*}{$\begin{array}{l}\text { PsA } \\
n=50 \\
(48 \%)\end{array}$} & \multirow{2}{*}{$\begin{array}{l}\text { PsO } \\
n=12(11 \\
\%)\end{array}$} & \multirow{2}{*}{$\begin{array}{l}\text { Hand OA } \\
n=13(12 \\
\%)\end{array}$} \\
\hline & & & \\
\hline SPARCC $(0-16)$ & $\begin{array}{l}2.0 \\
{[1.0 ; 5.0]}\end{array}$ & $\begin{array}{l}1.0 \\
{[0.0 ; 2.0]}\end{array}$ & $\begin{array}{l}2.0 \\
{[0.0 ; 2.0]}\end{array}$ \\
\hline NAPSI-score total finger $2(0-8)$ & $\begin{array}{l}3.0 \\
{[2.0 ; 4.0]}\end{array}$ & $\begin{array}{l}3.5 \\
{[2.0 ; 5.0]}\end{array}$ & $\begin{array}{l}0.0 \\
{[0.0 ; 0.0]}\end{array}$ \\
\hline NAPSI-score total finger $3(0-8)$ & $\begin{array}{l}3.0 \\
{[2.0 ; 4.0]}\end{array}$ & $\begin{array}{l}4.0 \\
{[2.3 ; 4.8]}\end{array}$ & $\begin{array}{l}0.0 \\
{[0.0 ; 0.0]}\end{array}$ \\
\hline NAPSI-score total finger $4(0-8)$ & $\begin{array}{l}2.0 \\
{[1.0 ; 4.0]}\end{array}$ & $\begin{array}{l}3.5 \\
{[2.0 ; 5.8]}\end{array}$ & $\begin{array}{l}0.0 \\
{[0.0 ; 0.0]}\end{array}$ \\
\hline NAPSI-score total finger $5(0-8)$ & $\begin{array}{l}2.0 \\
{[1.0 ; 4.0]}\end{array}$ & $\begin{array}{l}2.0 \\
{[1.0 ; 3.0]}\end{array}$ & $\begin{array}{l}0.0 \\
{[0.0 ; 0.0]}\end{array}$ \\
\hline
\end{tabular}

\section{Comparison of US, MRI and X-ray findings of the DIP joint and enthesis in PsA, PsO and OA}

The distribution of US outcomes of the three groups is shown in Fig. 2. The PsA group showed the highest prevalence of extensor tendon enthesitis (42.5\%), peritendonitis (13\%), and DIP joint erosions (11\%). The OA group had the highest prevalence of DIP joint SH (65.4\%), US Doppler (9.6\%) and NBF (86.5\%). Differences in US findings of the SEC are highlighted in Fig. 4.

The OA group presented the highest prevalence of all the outcomes in MRI PsAMRIS of the three groups and had no grade 0 outcomes in X-ray entheseal bone-change or erosions, as seen in Fig. 3. The PsA and PsO groups were similar in MRI PsAMRIS- and X-ray outcomes, but the PsA group presented more severe disease grades in both MRI DIP-joint synovitis score, MRI bone marrow oedema and a higher prevalence of MRI DIP-joint erosion.

The quantitative differences of US, MRI and X-ray, are shown in Table 2. US NBF in OA was statistically significantly different from PsA with a mean difference of 0.91 points $(95 \% \mathrm{Cl} 0.67$ to 1.16$)$ on the $0-3$ scale. OA was also statistically significantly different from PsA with a higher degree of US SH (mean difference: 0.50 points $(95 \% \mathrm{Cl} 0.21$ to 0.79$))$ on the $0-3$ scale.

Apart from dynamic contrast enhanced (DCE)-MRI measures of flow, the OA group was significantly different from PsA and PsO on all MRI PsAMRIS outcomes and X-ray outcomes (Table 2). These differences resulted in a much lower risk of having PsA than OA when these outcomes were present at a high grade in MRI PsAMRIS and X-ray, as shown in Table 3. In US, only the presence of Doppler signals and the severity of NBF were associated with a reduced risk of PsA, Risk Ratio 0.21 (CI95\% 0.06 to 0.75 ) and 0.52 (Cl95\% 0.43 to 0.63$)$ respectively. 
Table 2

Differences in MRI, X-ray and US outcomes of the distal interphalangeal joints and components of the synovial-entheseal-complex in PsA compared to OA and PsO with corresponding $\mathrm{Cl} 95 \%$. Abbreviations:

US: ultrasound. MRI: Magnetic resonance imaging, IRE: Initial rate of enhancement, ME: Maximum enhancement, Nvoxels: Number of enhancing voxels, DCE: Dynamic contrast enhanced, PsA: psoriatic

arthritis, PsO: skin psoriasis, OA: osteoarthritis. SE: standard error, mm.: millimetre.*: differences in binominal outcomes calculated using Pearson Chi-square.

\begin{tabular}{|c|c|c|c|c|c|c|}
\hline & $\begin{array}{l}\text { PsA mean } \\
\text { (SE) }\end{array}$ & Diagnosis & $\begin{array}{l}\text { Mean } \\
(\mathrm{SE})\end{array}$ & $\begin{array}{l}\text { Mean } \\
\text { difference }\end{array}$ & $\begin{array}{l}95 \% \\
\text { Confidence } \\
\text { interval }\end{array}$ & $\begin{array}{l}\mathrm{P}- \\
\text { value }\end{array}$ \\
\hline \multirow[t]{2}{*}{$\begin{array}{l}\text { US Extensor tendon } \\
\text { thickness, mm }\end{array}$} & $\begin{array}{l}0.669 \\
(0.013)\end{array}$ & OA & $\begin{array}{l}0.673 \\
(0.025)\end{array}$ & -0.004 & $\begin{array}{l}-0.059 \text { to } \\
0.051\end{array}$ & 0.88 \\
\hline & & $\mathrm{PsO}$ & $\begin{array}{l}0.682 \\
(0.027)\end{array}$ & -0.013 & $\begin{array}{l}-0.072 \text { to } \\
0.056\end{array}$ & 0.67 \\
\hline \multirow[t]{2}{*}{$\begin{array}{l}\text { US Flexor Tendon } \\
\text { thickness, mm }\end{array}$} & $\begin{array}{l}0.846 \\
(0.022)\end{array}$ & OA & $\begin{array}{l}0.888 \\
(0.043)\end{array}$ & -0.042 & $\begin{array}{l}-0.138 \text { to } \\
0.054\end{array}$ & 0.39 \\
\hline & & $\mathrm{PsO}$ & $\begin{array}{l}0.871 \\
(0.047)\end{array}$ & -0.025 & $\begin{array}{l}-0.128 \text { to } \\
0.079\end{array}$ & 0.64 \\
\hline \multirow[t]{2}{*}{$\begin{array}{l}\text { US nail matrix } \\
\text { thickness, mm }\end{array}$} & $\begin{array}{l}2.061 \\
(0.055)\end{array}$ & OA & $\begin{array}{l}2.119 \\
(0.107)\end{array}$ & -0.058 & $\begin{array}{l}-0.297 \text { to } \\
0.181\end{array}$ & 0.630 \\
\hline & & $\mathrm{PsO}$ & $\begin{array}{l}2.016 \\
(0.117)\end{array}$ & 0.045 & $\begin{array}{l}-0.211 \text { to } \\
0.301\end{array}$ & 0.727 \\
\hline \multirow[t]{2}{*}{$\begin{array}{l}\text { US nail bed } \\
\text { thickness, mm }\end{array}$} & $\begin{array}{l}1.769 \\
(0.051)\end{array}$ & OA & $\begin{array}{l}1.704 \\
(0.1)\end{array}$ & 0.065 & $\begin{array}{l}-0.157 \text { to } \\
0.287\end{array}$ & 0.565 \\
\hline & & PsO & $\begin{array}{l}1.964 \\
(0.108)\end{array}$ & -0.195 & $\begin{array}{l}-0.432 \text { to } \\
0.042\end{array}$ & 0.106 \\
\hline \multirow[t]{2}{*}{$\begin{array}{l}\text { US nail thickness, } \\
\text { mm }\end{array}$} & $\begin{array}{l}0.631 \\
(0.014)\end{array}$ & OA & $\begin{array}{l}0.612 \\
(0.027)\end{array}$ & 0.019 & $\begin{array}{l}-0.041 \text { to } \\
0.080\end{array}$ & 0.523 \\
\hline & & PsO & $\begin{array}{l}0.673 \\
(0.029)\end{array}$ & -0.042 & $\begin{array}{l}-0.106 \text { to } \\
0.023\end{array}$ & 0.202 \\
\hline \multirow[t]{2}{*}{ US flow Max ratio } & $\begin{array}{l}0.329 \\
(0.024)\end{array}$ & OA & $\begin{array}{l}0.338 \\
(0.047)\end{array}$ & -0.009 & $\begin{array}{l}-0.113 \text { to } \\
0.095\end{array}$ & 0.863 \\
\hline & & $\mathrm{PsO}$ & $\begin{array}{l}0.389 \\
(0.051)\end{array}$ & -0.060 & $\begin{array}{l}-0.172 \text { to } \\
0.051\end{array}$ & 0.287 \\
\hline \multirow[t]{2}{*}{ US flow Min ratio } & $\begin{array}{l}0.148 \\
(0.017)\end{array}$ & OA & $\begin{array}{l}0.121 \\
(0.034)\end{array}$ & 0.027 & $\begin{array}{l}-0.048 \text { to } \\
0.102\end{array}$ & 0.481 \\
\hline & & $\mathrm{PsO}$ & $\begin{array}{l}0.196 \\
(0.037)\end{array}$ & -0.049 & $\begin{array}{l}-0.129 \text { to } \\
0.032\end{array}$ & 0.231 \\
\hline $\begin{array}{l}\text { US synovial } \\
\text { hypertrophy }(0-3)\end{array}$ & $\begin{array}{l}0.520 \\
(0.067)\end{array}$ & OA & $\begin{array}{l}1.019 \\
(0.130)\end{array}$ & -0.499 & $\begin{array}{l}-0.790 \text { to } \\
-0.209\end{array}$ & 0.001 \\
\hline
\end{tabular}




\begin{tabular}{|c|c|c|c|c|c|c|}
\hline & $\begin{array}{l}\text { PsA mean } \\
\text { (SE) }\end{array}$ & Diagnosis & $\begin{array}{l}\text { Mean } \\
\text { (SE) }\end{array}$ & $\begin{array}{l}\text { Mean } \\
\text { difference }\end{array}$ & $\begin{array}{l}95 \% \\
\text { Confidence } \\
\text { interval }\end{array}$ & $\begin{array}{l}\mathrm{P}- \\
\text { value }\end{array}$ \\
\hline & & $\mathrm{PsO}$ & $\begin{array}{l}0.318 \\
(0.142)\end{array}$ & 0.202 & $\begin{array}{l}-0.109 \text { to } \\
0.513\end{array}$ & 0.201 \\
\hline \multirow[t]{2}{*}{$\begin{array}{l}\text { US Doppler signals } \\
(0-3)\end{array}$} & $\begin{array}{l}0.030 \\
(0.021)\end{array}$ & OA & $\begin{array}{l}0.135 \\
(0.047)\end{array}$ & -0.105 & $\begin{array}{l}-0.194 \text { to } \\
-0.015\end{array}$ & 0.023 \\
\hline & & $\mathrm{PsO}$ & $\begin{array}{l}0.045 \\
(0.044)\end{array}$ & 0.030 & $\begin{array}{l}-0.111 \text { to } \\
0.081\end{array}$ & 0.750 \\
\hline \multirow[t]{2}{*}{ US erosions (0-3) } & $\begin{array}{l}0.140 \\
(0.027)\end{array}$ & OA & $\begin{array}{l}0.096 \\
(0.053)\end{array}$ & 0.044 & $\begin{array}{l}-0.073 \text { to } \\
0.161\end{array}$ & 0.460 \\
\hline & & PsO & $\begin{array}{l}0.023 \\
(0.057)\end{array}$ & 0.117 & $\begin{array}{l}-0.008 \text { to } \\
0.243\end{array}$ & 0.067 \\
\hline \multirow[t]{2}{*}{$\begin{array}{l}\text { US new bone } \\
\text { formation }(0-3)\end{array}$} & $\begin{array}{l}0.530 \\
(0.056)\end{array}$ & OA & $\begin{array}{l}1.442 \\
(0.110)\end{array}$ & -0.912 & $\begin{array}{l}-1.158 \text { to } \\
-0.667\end{array}$ & $\begin{array}{l}<.001 \\
0.001\end{array}$ \\
\hline & & $\mathrm{PsO}$ & $\begin{array}{l}0.295 \\
(0.120)\end{array}$ & 0.235 & $\begin{array}{l}-0.028 \text { to } \\
0.497\end{array}$ & 0.079 \\
\hline \multirow[t]{2}{*}{$\begin{array}{l}\text { MRI synovial } \\
\text { hypertrophy }\end{array}$} & $\begin{array}{l}0.482 \\
(0.066)\end{array}$ & OA & $\begin{array}{l}1.271 \\
(0.123)\end{array}$ & -0.789 & $\begin{array}{l}-1.067 \text { to } \\
0.510\end{array}$ & $\begin{array}{l}<.001 \\
0.00\end{array}$ \\
\hline & & $\mathrm{PsO}$ & $\begin{array}{l}0.389 \\
(0.142)\end{array}$ & 0.093 & $\begin{array}{l}-0.219 \text { to } \\
0.406\end{array}$ & 0.553 \\
\hline \multirow[t]{2}{*}{ MRI tenosynovitis } & $\begin{array}{l}0.381 \\
(0.060)\end{array}$ & OA & $\begin{array}{l}0.833 \\
(0.113)\end{array}$ & -0.452 & $\begin{array}{l}-0.709 \text { to } \\
-0.196\end{array}$ & 0.001 \\
\hline & & $\mathrm{PsO}$ & $\begin{array}{l}0.500 \\
(0.131)\end{array}$ & -0.119 & $\begin{array}{l}-0.407 \text { to } \\
0.169\end{array}$ & 0.441 \\
\hline \multirow[t]{2}{*}{$\begin{array}{l}\text { MRI periarticular } \\
\text { inflammation }\end{array}$} & $\begin{array}{l}0.673 \\
(0.755)\end{array}$ & OA & $\begin{array}{l}1.563 \\
(0.140)\end{array}$ & -0.890 & $\begin{array}{l}-1.206 \text { to } \\
-0.573\end{array}$ & $\begin{array}{l}<.001 \\
0.00\end{array}$ \\
\hline & & $\mathrm{PsO}$ & $\begin{array}{l}0.725 \\
(0.153)\end{array}$ & -0.052 & $\begin{array}{l}-0.393 \text { to } \\
0.288\end{array}$ & 0.759 \\
\hline \multirow[t]{2}{*}{$\begin{array}{l}\text { MRI bone marrow } \\
\text { oedema }\end{array}$} & $\begin{array}{l}0.387 \\
(0.138)\end{array}$ & $O A$ & $\begin{array}{l}1.917 \\
(0.259)\end{array}$ & -1.530 & $\begin{array}{l}-0.157 \text { to } \\
0.287\end{array}$ & $\begin{array}{l}< \\
0.001\end{array}$ \\
\hline & & $\mathrm{PsO}$ & $\begin{array}{l}0.250 \\
(0.270)\end{array}$ & 0.137 & $\begin{array}{l}-0.470 \text { to } \\
0.744\end{array}$ & 0.654 \\
\hline \multirow[t]{2}{*}{ MRI erosions } & $\begin{array}{l}0.197 \\
(0.069)\end{array}$ & OA & $\begin{array}{l}1.271 \\
(0.129)\end{array}$ & -1.074 & $\begin{array}{l}-1.365 \text { to } \\
-0.782\end{array}$ & $\begin{array}{l}<.001 \\
0.001\end{array}$ \\
\hline & & $\mathrm{PsO}$ & $\begin{array}{l}0.091 \\
(0.134)\end{array}$ & 0.106 & $\begin{array}{l}-0.195 \text { to } \\
0.408\end{array}$ & 0.484 \\
\hline $\begin{array}{l}\text { DCE-MRI flow } \\
\text { ME*nVOXEL }\end{array}$ & $\begin{array}{l}1062.886 \\
(110.177)\end{array}$ & OA & $\begin{array}{l}879.622 \\
(193.744)\end{array}$ & 183.264 & $\begin{array}{l}-247.983 \text { to } \\
614.510\end{array}$ & 0.399 \\
\hline
\end{tabular}




\begin{tabular}{|c|c|c|c|c|c|c|}
\hline & $\begin{array}{l}\text { PsA mean } \\
\text { (SE) }\end{array}$ & Diagnosis & $\begin{array}{l}\text { Mean } \\
(\mathrm{SE})\end{array}$ & $\begin{array}{l}\text { Mean } \\
\text { difference }\end{array}$ & $\begin{array}{l}95 \% \\
\text { Confidence } \\
\text { interval }\end{array}$ & $\begin{array}{l}\mathrm{P} \text { - } \\
\text { value }\end{array}$ \\
\hline & & $\mathrm{PsO}$ & $\begin{array}{l}699.788 \\
(202.359)\end{array}$ & 363.098 & $\begin{array}{l}-83.617 \text { to } \\
809.813\end{array}$ & 0.109 \\
\hline \multirow[t]{2}{*}{$\begin{array}{l}\text { DCE-MRI flow } \\
\text { IRE*nVOXEL }\end{array}$} & $\begin{array}{l}8.647 \\
(0.983)\end{array}$ & OA & $\begin{array}{l}5.322 \\
(1.966)\end{array}$ & 3.325 & $\begin{array}{l}-1.073 \text { to } \\
7.722\end{array}$ & 0.136 \\
\hline & & $\mathrm{PsO}$ & $\begin{array}{l}11.042 \\
(2.313)\end{array}$ & -2.395 & $\begin{array}{l}-7.422 \text { to } \\
2.632\end{array}$ & 0.344 \\
\hline \multirow[t]{2}{*}{ X-ray erosions $(0-3)$} & $\begin{array}{l}1.163 \\
(0.091)\end{array}$ & OA & $\begin{array}{l}2.077 \\
(0.177)\end{array}$ & -0.914 & $\begin{array}{l}-1.310 \text { to } \\
-0.418\end{array}$ & $\hat{0} .001$ \\
\hline & & $\mathrm{PsO}$ & $\begin{array}{l}0.917 \\
(0.184)\end{array}$ & 0.247 & $\begin{array}{l}-0.162 \text { to } \\
0.656\end{array}$ & 0.233 \\
\hline \multirow[t]{2}{*}{$\begin{array}{l}\text { X-ray entheseal } \\
\text { change }(0-3)\end{array}$} & $\begin{array}{l}1.255 \\
(0.081)\end{array}$ & OA & $\begin{array}{l}1.731 \\
(0.158)\end{array}$ & -0.476 & $\begin{array}{l}-0.830 \text { to } \\
-0.121\end{array}$ & 0.009 \\
\hline & & $\mathrm{PsO}$ & $\begin{array}{l}0.833 \\
(0.164)\end{array}$ & 0.422 & $\begin{array}{l}0.056 \text { to } \\
0.788\end{array}$ & 0.024 \\
\hline
\end{tabular}


Table 3

The odds ratio and risk ratio for having psoriatic arthritis if imaging outcomes is present in MRI, X-ray and US compared to PsO or OA with corresponding $95 \% \mathrm{Cl}$. Abbreviations: MRI: magnetic resonance imaging, US: ultrasound, PsA: psoriatic arthritis, PsO: skin psoriasis, OA: hand osteoarthritis. *: p-value for odds ratio calculated by Mantel-Haenszel Common Odds Ratio Estimate.

\begin{tabular}{|c|c|c|c|c|c|}
\hline \multirow{2}{*}{$\begin{array}{l}\text { Imaging outcomes } \\
\text { MRI DIP-joint } \\
\text { synovial hypertrophy }\end{array}$} & \multicolumn{2}{|c|}{ Groups n(\%)/n(\%) } & \multirow{2}{*}{ 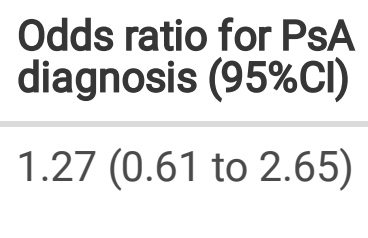 } & \multirow{2}{*}{$\begin{array}{l}\begin{array}{l}\text { p- } \\
\text { value* }\end{array} \\
0.528\end{array}$} & \multirow{2}{*}{$\begin{array}{l}\begin{array}{l}\text { Risk ratio fol } \\
\text { PsA }(95 \% \mathrm{Cl})\end{array} \\
1.15(0.74 \text { to } \\
1.79)\end{array}$} \\
\hline & $\begin{array}{l}\text { PsA / } \\
\text { PsO }\end{array}$ & $\begin{array}{l}75(45 \%) / 14 \\
(39 \%)\end{array}$ & & & \\
\hline & $\begin{array}{l}\text { PSA / } \\
\text { OA }\end{array}$ & $\begin{array}{l}75(45 \%) / 42 \\
(88 \%)\end{array}$ & 0.12 (0.05 to 0.29$)$ & $\hat{0.001}$ & $\begin{array}{l}0.51(0.42 \text { to } \\
0.62)\end{array}$ \\
\hline \multirow[t]{2}{*}{ MRI Tenosynovitis } & $\begin{array}{l}\text { PsA / } \\
\text { PsO }\end{array}$ & $\begin{array}{l}58(35 \%) / 18 \\
(50 \%)\end{array}$ & 0.53 (0.26 to 1.09$)$ & 0.081 & $\begin{array}{l}0.69 \text { (0.47 to } \\
1.02)\end{array}$ \\
\hline & $\begin{array}{l}\text { PSA / } \\
\text { OA }\end{array}$ & $\begin{array}{l}558(35 \\
\%) / 35(73 \%)\end{array}$ & 0.20 (0.10 to 0.40$)$ & $\hat{0} .001$ & $\begin{array}{l}0.47(0.36 \text { to } \\
0.62)\end{array}$ \\
\hline \multirow[t]{2}{*}{$\begin{array}{l}\text { MRI New bone } \\
\text { formation }\end{array}$} & $\begin{array}{l}\text { PsA / } \\
\text { PsO }\end{array}$ & $\begin{array}{l}101(60 \\
\%) / 27(61 \%)\end{array}$ & 0.95 (0.48 to 1.88$)$ & 0.881 & $\begin{array}{l}0.98(0.75 \text { to } \\
1.28)\end{array}$ \\
\hline & $\begin{array}{l}\text { PsA / } \\
\text { OA }\end{array}$ & $\begin{array}{l}101(60 \%) / 45 \\
(94 \%)\end{array}$ & 0.10 (0.03 to 0.34$)$ & $<.001$ & $\begin{array}{l}0.64(0.56 \text { to } \\
0.74)\end{array}$ \\
\hline \multirow[t]{2}{*}{$\begin{array}{l}\text { MRI Periarticular } \\
\text { inflammation }\end{array}$} & $\begin{array}{l}\text { PsA / } \\
\text { PsO }\end{array}$ & $\begin{array}{l}82(49 \%) / 24 \\
(60 \%)\end{array}$ & 0.64 (0.32 to 1.28$)$ & 0.205 & $\begin{array}{l}0.81 \text { ( } 0.61 \text { to } \\
1.09)\end{array}$ \\
\hline & $\begin{array}{l}\text { PsA / } \\
\text { OA }\end{array}$ & $\begin{array}{l}82(49 \%) / 43 \\
(90 \%)\end{array}$ & $0.11(0.04$ to 0.29$)$ & $\hat{0.001}$ & $\begin{array}{l}0.55(0.45 \text { to } \\
0.65)\end{array}$ \\
\hline \multirow[t]{2}{*}{$\begin{array}{l}\text { MRI Bone marrow } \\
\text { oedema }\end{array}$} & $\begin{array}{l}\text { PsA / } \\
\text { PsO }\end{array}$ & $\begin{array}{l}24(14 \%) / 7 \\
(16 \%)\end{array}$ & 0.88 (0.35 to 2.20$)$ & 0.786 & $\begin{array}{l}0.90(0.41 \text { to } \\
1.95)\end{array}$ \\
\hline & $\begin{array}{l}\text { PsA / } \\
\text { OA }\end{array}$ & $\begin{array}{l}24(14 \%) / 34 \\
(71 \%)\end{array}$ & 0.07 (0.03 to 0.15$)$ & $<.001$ & $\begin{array}{l}0.20(0.13 \text { to } \\
0.31)\end{array}$ \\
\hline \multirow[t]{2}{*}{ MRI Erosions } & $\begin{array}{l}\mathrm{PsA} / \\
\mathrm{PsO}\end{array}$ & $\begin{array}{l}25(15 \%) / 3 \\
(7 \%)\end{array}$ & 2.41 (0.69 to 8.37 ) & 0.168 & $\begin{array}{l}2.20(0.70 \text { to } \\
6.94)\end{array}$ \\
\hline & $\begin{array}{l}\text { PsA / } \\
\text { OA }\end{array}$ & $\begin{array}{l}25(15 \%) / 31 \\
(65 \%)\end{array}$ & 0.10 (0.05 to 0.20$)$ & <. 001 & $\begin{array}{l}0.23(0.15 \text { to } \\
0.35)\end{array}$ \\
\hline \multirow[t]{2}{*}{ X-ray Erosions } & $\begin{array}{l}\text { PsA / } \\
\text { PsO }\end{array}$ & $\begin{array}{l}156(80 \\
\%) / 37(77 \%)\end{array}$ & 1.16 (0.54 to 2.47$)$ & 0.702 & $\begin{array}{l}1.03 \text { (0.87 to } \\
1.22)\end{array}$ \\
\hline & $\begin{array}{l}\text { PsA / } \\
\text { OA }\end{array}$ & $\begin{array}{l}156(80 \\
\%) / 52(100 \\
\%)\end{array}$ & n.e. & - & $\begin{array}{l}0.80(0.74 \text { to } \\
0.85)\end{array}$ \\
\hline \multirow[t]{2}{*}{$\begin{array}{l}\text { X-ray Entheseal } \\
\text { change }\end{array}$} & $\begin{array}{l}\mathrm{PsA} / \\
\mathrm{PsO}\end{array}$ & $\begin{array}{l}167(85 \\
\%) / 38(80 \%)\end{array}$ & 1.52 (0.68 to 3.37$)$ & 0.309 & $\begin{array}{l}1.08(0.92 \text { to } \\
1.26)\end{array}$ \\
\hline & $\begin{array}{l}\text { PsA / } \\
\text { OA }\end{array}$ & $\begin{array}{l}167(85 \\
\%) / 52(100 \\
\%)\end{array}$ & n.e. & - & $\begin{array}{l}0.85(0.80 \text { to } \\
0.90)\end{array}$ \\
\hline
\end{tabular}




\begin{tabular}{|c|c|c|c|c|c|}
\hline \multirow{2}{*}{$\begin{array}{l}\text { Imaging outcomes } \\
\text { US DIP-joint synovial } \\
\text { hypertrophy }\end{array}$} & \multicolumn{2}{|c|}{ Groups n(\%)/n(\%) } & \multirow{2}{*}{$\begin{array}{l}\text { Odds ratio for PsA } \\
\text { diagnosis (95\% }\end{array}$} & \multirow{2}{*}{$\begin{array}{l}\text { p- } \\
\text { value* } \\
0.057\end{array}$} & \multirow{2}{*}{$\begin{array}{l}\text { Risk ratio fo } \\
\text { PsA (95\%Cl) } \\
1.58(0.95 \text { to } \\
2.62)\end{array}$} \\
\hline & $\begin{array}{l}\text { PsA / } \\
\text { PsO }\end{array}$ & $\begin{array}{l}86(43 \%) / 12 \\
(27 \%)\end{array}$ & & & \\
\hline & $\begin{array}{l}\text { PsA / } \\
\text { OA }\end{array}$ & $\begin{array}{l}86(43 \%) / 34 \\
(65 \%)\end{array}$ & 0.40 (0.21 to 0.76$)$ & 0.005 & $\begin{array}{l}0.66(0.51 \text { to } \\
0.85)\end{array}$ \\
\hline \multirow[t]{2}{*}{ US Doppler signals } & $\begin{array}{l}\text { PsA / } \\
\text { PsO }\end{array}$ & $\begin{array}{l}4(2 \%) / 1(2 \\
\%)\end{array}$ & 0.88 (0.10 to 8.05$)$ & 0.908 & $\begin{array}{l}0.88(0.10 \text { to } \\
7.68)\end{array}$ \\
\hline & $\begin{array}{l}\text { PsA / } \\
\text { OA }\end{array}$ & $\begin{array}{l}4(2 \%) / 5(10 \\
\%)\end{array}$ & 0.20 (0.10 to 0.74$)$ & 0.017 & $\begin{array}{l}0.21(0.06 \text { to } \\
0.75)\end{array}$ \\
\hline \multirow[t]{2}{*}{ US Erosions } & $\begin{array}{l}\text { PsA / } \\
\text { PsO }\end{array}$ & $\begin{array}{l}24(12 \%) / 1 \\
(2 \%)\end{array}$ & 5.86 (0.77 to 44.56$)$ & 0.087 & $\begin{array}{l}5.28(0.73 \text { to } \\
38.00)\end{array}$ \\
\hline & $\begin{array}{l}\text { PsA / } \\
\text { OA }\end{array}$ & $\begin{array}{l}24(12 \%) / 5 \\
(10 \%)\end{array}$ & 1.28 (0.46 to 3.54$)$ & 0.632 & $\begin{array}{l}1.25(0.50 \text { to } \\
3.11)\end{array}$ \\
\hline \multirow[t]{2}{*}{$\begin{array}{l}\text { US New bone } \\
\text { formation }\end{array}$} & $\begin{array}{l}\text { PsA / } \\
\text { PsO }\end{array}$ & $\begin{array}{l}90(45 \%) / 13 \\
(30 \%)\end{array}$ & 1.95 (0.96 to 3.95$)$ & 0.063 & $\begin{array}{l}1.52(0.25 \text { to } \\
2.47)\end{array}$ \\
\hline & $\begin{array}{l}\text { PsA / } \\
\text { OA }\end{array}$ & $\begin{array}{l}90(45 \%) / 45 \\
(87 \%)\end{array}$ & $0.13(0.06$ to 0.30$)$ & $\langle .001$ & $\begin{array}{l}0.52(0.43 \text { to } \\
0.63)\end{array}$ \\
\hline \multirow[t]{2}{*}{$\begin{array}{l}\text { US Extensor tendon } \\
\text { enthesitis }\end{array}$} & $\begin{array}{l}\text { PsA / } \\
\text { PsO }\end{array}$ & $\begin{array}{l}85(43 \%) / 17 \\
(39 \%)\end{array}$ & 1.17 (0.60 to 2.29$)$ & 0.638 & $\begin{array}{l}1.10(0.73 \text { to } \\
1.65)\end{array}$ \\
\hline & $\begin{array}{l}\text { PsA / } \\
\text { OA }\end{array}$ & $\begin{array}{l}85(43 \%) / 15 \\
(29 \%)\end{array}$ & 1.82 (0.94 to 3.54$)$ & 0.075 & $\begin{array}{l}1.48(0.93 \text { to } \\
2.33)\end{array}$ \\
\hline \multirow[t]{2}{*}{ US Peritendonitis } & $\begin{array}{l}\text { PsA / } \\
\text { PsO }\end{array}$ & $\begin{array}{l}26(13 \%) / 2 \\
(5 \%)\end{array}$ & 3.14 (0.72 to 13.75$)$ & 0.111 & $\begin{array}{l}2.86(0.71 \text { to } \\
11.61)\end{array}$ \\
\hline & $\begin{array}{l}\text { PSA / } \\
\text { OA }\end{array}$ & $\begin{array}{l}26(13 \%) / 4 \\
(8 \%)\end{array}$ & 1.79 (0.60 to 5.39$)$ & 0.298 & $\begin{array}{l}1.69(0.62 \text { to } \\
4.63)\end{array}$ \\
\hline
\end{tabular}

PsA and PsO were differentiated by the presence of erosions and NBF with a mean difference of 0.12 points $(95 \% \mathrm{Cl}-0.01$ to $0.24, \mathrm{p}=0.07$ ) and mean 0.24 points $(95 \% \mathrm{Cl}-0.03$ to $0.50, \mathrm{p}=0.08)$, respectively, on 0-3 scales. A more pronounced difference in the presence of entheseal change between PsA and PsO was found in X-ray 0.42 points $(95 \% \mathrm{Cl} 0.06$ to $0.79, \mathrm{p}=0.024)$. No outcome in US, MRI or X-ray was associated with a higher risk of having PsA versus PsO (Table 3 ).

No measures of flow in US Doppler quantification or DCE-MRI could differentiate between PSA, PsO or OA. Numeric measures of the flexor-/extensor enthesis, nail bed/matrix or nail did not reveal any significant outcome patterns in the three groups, which was reflected in an overall low sensitivity to PsA diagnosis shown in Supplementary Table S8. The best discriminatory factor between PsA versus PsO was DCE-MRI Flow ME*nVOXEL $0.59(95 \% \mathrm{Cl} 0.47$ to 0.68$)$ and PsA versus OA DCE-MRI Flow IRE*nVOXEL 0.65 (95\% Cl 0.56 to 0.74 ). 


\section{Discussion}

In this comprehensive cross-sectional study, we demonstrated significant patterns of US, MRI and X-ray findings dependent on the underlying diagnosis. Using US, the PSA group was differentiated from the other groups by the presence of extensor tendon enthesitis, erosions and peritendonitis (Fig. 2). Apart from X-ray detected entheseal change, it was not possible to differentiate between the PsA group and PsO group using MRI or X-ray, thus strengthening the link of nail disease to enthesitis and synovitis as a continuum with a yet unknown threshold (33). Nevertheless, differences in PsA and PsO patients was identified looking at structural disease grade in both US and MRI outcomes (Figs. 2 and 3). In PsO patients, the diagnosis of PsA is not likely unless they present with X-ray enthesitis and at least a grade 2 US erosion and US NBF score or grade 2 MRI synovitis-, tenosynovitis-, erosion score or grade 3 MRI bone marrow oedema.

A high prevalence of US NBF, Doppler and SH were seen in patients with OA of the DIP joints, and these patients also reported the highest 68/66 tender joint score, VAS joint-related pain, and HAQ-DI (Table 1). It was surprising that the OA group also exhibited US enthesitis, but it has been proposed that PsA and OA could share a common enthesis-associated area with microdamage as a common disease driver (34). It could also reflect that we cannot differentiate capsular hypertrophy and enthesitis with our current imaging resolution as it may look the same on US and MRI. US only revealed subtle differences between the PsA and OA group, but MRI PsAMRIS and X-ray revealed considerable differences in disease severity in all semiquantitative outcomes. A high prevalence of these findings reduces the risk of PsA (Table 3 ). This opposes the notion that DIP-joint synovitis is more severe in autoimmune processes $(35,36)$ and could reflect that the OA group does not receive adequate treatment. In the PsA group, these symptoms are likely suppressed as most receive DMARD treatment.

No US Doppler was observed in the flexor or extensor enthesis in either patient group, and caution should be exhibited because of greater flow from adjacent dorsal branches from lateral digital arteries might obscure any smaller neoangiogenic vessels in the enthesis. In this context, using a Doppler signal $\leq 2 \mathrm{~mm}$ from the bony cortex as a landmark of enthesitis in SpA as suggested in Outcome Measures in Rheumatology (37) may not be reliable for the extensor tendon at the DIP-joint.

Even though DCE-MRI Flow ME*nVOXEL and IRE*nVOXEL proved to have the highest probability of differentiating between PsA versus PsO and PsA versus OA, it did not prove sensitive enough to be of diagnostic use in the current study, but this could be due to the relative small sample size of OA and PsO patients warranting larger studies to study this association in more details. To support the current findings Schraml et al. (38) also found comparable synovial enhancement between PsA and OA until 15 minutes post-contrast, where higher values were found in OA. The same group also found a difference between RA and PsA 15 minutes post-contrast, but a correlation between clinical findings and DCE-MRI only existed in the RA group (39). We did not obtain data 15 minutes post-contrast because of an inherent overestimation of enhancement of synovitis on static postcontrast enhanced images based on the risk of diffusion of contrast to adjacent extracellular fluid already after 5 minutes post contrast injection (40). 


\section{Strengths}

The study benefits from consecutively enrolled patients in outpatient clinics in dermatology and rheumatology comparing PsA and PsO patients already in DMARD treatment, reflecting the diagnostic dilemmas in a clinical setting, thus strengthening the external validity. The imaging assessors were blinded to the diagnose and results from the other findings.

\section{Limitations}

A consecutive enrollment strategy resulted in an all-female hand OA group, which might weaken the generalisability of our findings. However, the Framingham Studies showed a higher prevalence of hand OA among women $(41,42)$.

A possible limitation is that a high proportion of the PsA and PsO patients were in DMARD therapy. This is likely to lower the chance of findings related to inflammatory activity. It may also explain the relatively high prevalence of these findings in the OA group as they are not offered DMARD therapy.

The cross-sectional design limits any conclusions about temporal developments and association over time of the different findings.

\section{Conclusions}

In conclusion, we found that the differentiation between PsA, PsO, and hand OA using US, MRI, and X-ray is possible based on the degree of structural involvement using a combination of semiquantitative OMERACT US scores, PSAMRIS score and X-ray score. With a high grade of US, MRI and X-ray NBF and MRI bone marrow oedema, the OA diagnosis is more likely than PsA. In demarcating PsA from PsO patients, it is of significance if they present with X-ray enthesitis and a high degree of US erosions and NBF or MRI synovitis-, tenosynovitis-, erosion score or bone marrow oedema.

Quantitative measures of flow in both DCE-MRI and US and US measures of DIP-joint tendon enthesis, nail and nailbed/matrix are of limited value in differentiating between PsA, PsO and OA. X-ray can be used to identify hand OA but not to distinguish between PsA and PsO.

\section{Abbreviations}

AUC Area under the curve

bDMARD biological Disease Modifying Anti-Rheumatic Drug

BMI Body mass index

csDMARD Conventional Disease Modifying Anti-Rheumatic Drug

DCE Dynamic contrast enhanced 
DIP Distal interphalangeal

HAQ-DI Health Assessment Questionnaire disability index

ICC Intraclass correlation coefficient

IRE Initial rate of enhancement

ME Maximum enhancement

MRI Magenetic resonance imaging

NAPSI Nail Psoriasis Severity Index

NBF New bone formation

Nvoxels number of enhancing voxels

OA Hand osteoarthritis

PASI Psoriatic Area Severity Index

PROM Patient-reported outcome measures

PsA Psoriatic arthritis

PsO Skin psoriasis

ROI Region of interest

RR Risk ratio

SEC Synovio-entheseal complex

SPARCC Spondyloarthritis Research Consortium of Canada

US Ultrasound

VAS Visial analog scale

\section{Declarations}

\section{Ethics approval and consent to participate}

This cross-sectional study was approved by the Ethics Committee on 25-05-2018, Journal-nr.: H18006696 and the Danish Data Protection Agency on 04-06-2018 (VD-2018-149, med I-Suite nr.: 6392). 


\section{Consent for publication}

Consent for data sharing was not obtained, but no individual person's data is included in the manuscript. The dataset is anonymised, and the risk of identification is very low.

\section{Availability of data and materials}

The dataset will be available from the corresponding author to the extent possible under the Danish national law.

\section{Competing interests}

JGM has received speaking fees from AbbVie, NOVARTIS, Eli-Lilly and BK Ultrasound outside the present work. LEK has received fees for speaking and/or consultancy from Pfizer, AbbVie, Amgen, UCB, Celgene, BMS, Biogen, Sanofi, MSD, Novartis, Eli Lilly, Janssen Pharmaceuticals. MH declares to be a member of the scientific advisory board of the Thuasne group. MB has received speaking fee from Abbvie, Novartis, Esaote, Eli-Lilly, UCB and is a consultant for Image Analysis Group. LD has received speaking fees from UCB, MSD, Eli-Lilly, Janssen Pharmaceuticals, and a BMS research grant outside the present work. ALT has acted as a consultant for or received honoraria from Abbvie, Eli Lilly, Gilead, Janssen, Novartis, Pfizer, UCB. OK and JD are shareholders and employees at IAG, Image Analysis Group. KE, RJW and MM declare no competing interests.

\section{Funding}

The study was funded by an investigator-initiated grant from Novartis, a core grant from The Oak Foundation (OCAY-13-309) and the Minister Erna Hamilton Foundation for Science and Art.

\section{Authors' contributions}

JGM, MH and LEK had full access to all the data in the study and take responsibility for the integrity of the data and the accuracy of the data analysis. JGM, LEK: study concept and design. JGM, KE, MM, MB: acquisition of data. JGM, MH and LEK: statistical analysis. All authors: interpretation of data; critical revision of the manuscript for important intellectual content. JGM: drafting of the manuscript. $\mathrm{MH}, \mathrm{KE}$ and LEK: study supervision. 


\section{Acknowledgements}

We wish to thank René Cordtz at the Department of Clinical Medicine, University of Aalborg, Denmark for proofreading and Line Rustad at the Parker Institute for organising the flow of participants. A huge thanks to Lene Terslev, Hilde Hammer, Heidi Siddle, Ruth Wittoek, Kate Smith, Etienne Qvistgaard, Lene Jensen, Lars Juul and Marion Kortekaas for contributing to the preliminary probe comparison web-survey. A huge thanks to MRI staff for performing the examinations, with a special thanks to Janus Damm Nybing and Frederik Hvid Linden.

\section{References}

1. O’Neill T, Silman AJ. Psoriatic arthritis. Historical background and epidemiology. Baillieres Clin Rheumatol. 1994 May;8(2):245-61.

2. Tillett W, Charlton R, Nightingale A, Snowball J, Green A, Smith C, et al. Interval between onset of psoriasis and psoriatic arthritis comparing the UK Clinical Practice Research Datalink with a hospitalbased cohort. Rheumatol (United Kingdom). 2017;56(12):2109-13.

3. Villani AP, Rouzaud M, Sevrain M, Barnetche T, Paul C, Richard MA, et al. Prevalence of undiagnosed psoriatic arthritis among psoriasis patients: Systematic review and meta-analysis. J Am Acad Dermatol. 2015;73(2):242-8.

4. Egeberg A, Kristensen LE, Thyssen JP, Gislason GH, Gottlieb AB, Coates LC, et al. Incidence and prevalence of psoriatic arthritis in Denmark: a nationwide register linkage study. Ann Rheum Dis. 2017 Sep;76(9):1591-7.

5. Gladman DD, Antoni C, Mease P, Clegg DO, Nash P, Nash O. Psoriatic arthritis: Epidemiology, clinical features, course, and outcome. In: Annals of the Rheumatic Diseases. 2005. p. ii14-7.

6. Lee S, Mendelsohn A, Sarnes E. The burden of psoriatic arthritis: a literature review from a global health systems perspective. P T. 2010 Dec;35(12):680-9.

7. Mease PJ. Psoriatic arthritis: update on pathophysiology, assessment and management. Ann Rheum Dis [Internet]. 2011 Mar 1 [cited 2017 Jun 26];70 Suppl 1(Suppl 1):i77-84. Available from: http://ard.bmj.com/cgi/doi/10.1136/ard.2010.140582.

8. Taylor W, Gladman D, Helliwell P, Marchesoni A, Mease P, Mielants H, et al. Classification criteria for psoriatic arthritis: development of new criteria from a large international study. Arthritis Rheum. 2006 Aug;54(8):2665-73.

9. Coates LC, Helliwell PS. Psoriatic arthritis: State of the art review. Vol. 17, Clinical Medicine, Journal of the Royal College of Physicians of London. 2017. p. 65-70.

10. Kristensen LE, Jørgensen TS, Christensen R, Gudbergsen H, Dreyer L, Ballegaard C, et al. Societal costs and patients' experience of health inequities before and after diagnosis of psoriatic arthritis: A Danish cohort study. Ann Rheum Dis. 2017;76(9):1495-501. 
11. Ballegaard C, Højgaard P, Dreyer L, Cordtz R, Jørgensen TS, Skougaard M, et al. Impact of Comorbidities on Tumor Necrosis Factor Inhibitor Therapy in Psoriatic Arthritis: A Population-Based Cohort Study. Arthritis Care Res. 2018.

12. Wiell C, Szkudlarek M, Hasselquist M, Møller JM, Vestergaard A, Nørregaard J, et al. Ultrasonography, magnetic resonance imaging, radiography, and clinical assessment of inflammatory and destructive changes in fingers and toes of patients with psoriatic arthritis. Arthritis Res Ther. 2007;9(6):R119.

13. Zhang W, Doherty M, Leeb BF, Alekseeva L, Arden NK, Bijlsma JW, et al. EULAR evidence-based recommendations for the diagnosis of hand osteoarthritis: report of a task force of ESCISIT. Ann Rheum Dis. 2009.

14. Tan AL, Grainger AJ, Tanner SF, Emery P, McGonagle D. A high-resolution magnetic resonance imaging study of distal interphalangeal joint arthropathy in psoriatic arthritis and osteoarthritis: are they the same? Arthritis Rheum. 2006 Apr;54(4):1328-33.

15. Love TJ, Gudjonsson JE, Valdimarsson H, Gudbjornsson B. Small joint involvement in psoriatic arthritis is associated with onycholysis: The reykjavik psoriatic arthritis study. Scand J Rheumatol. 2010;39(4):299-302.

16. Eder L, Chandran V, Shen H, Cook RJ, Shanmugarajah S, Rosen CF, et al. Incidence of arthritis in a prospective cohort of psoriasis patients. Arthritis Care Res (Hoboken). 2011;63(4):619-22.

17. Eder L, Law T, Chandran V, et al. Association between environmental factors and onset of psoriatic arthritis in patients with psoriasis. Vol. 63, Arthritis Care and Research. 2011. p. 1091-7.

18. Ogdie A, Weiss P. The Epidemiology of Psoriatic Arthritis. Vol. 41, Rheumatic Disease Clinics of North America. 2015. p. 545-68.

19. McGonagle D. Enthesitis: an autoinflammatory lesion linking nail and joint involvement in psoriatic disease. J Eur Acad Dermatol Venereol [Internet]. 2009 Sep [cited 2017 Jun 26];23 Suppl 1:9-13. Available from: http://doi.wiley.com/10.1111/j.1468-3083.2009.03363.x.

20. McGonagle D, Tan AL, Benjamin M. The nail as a musculoskeletal appendage-implications for an improved understanding of the link between psoriasis and arthritis. Dermatology [Internet]. 2009 Dec 6 [cited 2017 Jun 26];218(2):97-102. Available from: http://www.karger.com/? doi=10.1159/000182250.

21. Acosta-Felquer ML, Ruta S, Rosa J, Marin J, Ferreyra-Garrot L, Galimberti ML, et al. Ultrasound entheseal abnormalities at the distal interphalangeal joints and clinical nail involvement in patients with psoriasis and psoriatic arthritis, supporting the nail-enthesitis theory. Semin Arthritis Rheum [Internet]. 2017 May 11 [cited 2017 Aug 7]; Available from: http://linkinghub.elsevier.com/retrieve/pii/S0049017217302378.

22. Maejima $\mathrm{H}$, Taniguchi T, Watarai A, Katsuoka K. Evaluation of nail disease in psoriatic arthritis by using a modified nail psoriasis severity score index. Int J Dermatol. 2010;49(8):901-6.

23. Tehranzadeh J, Ashikyan O, Dascalos J, Dennehey C. MRI of large intraosseous lesions in patients with inflammatory arthritis. American Journal of Roentgenology. 2004. 
24. Backhaus M, Kamradt T, Sandrock D, Loreck D, Fritz J, Wolf KJ, et al. Arthritis of the finger joints: A comprehensive approach comparing conventional radiography, scintigraphy, ultrasound, and contrast-enhanced magnetic resonance imaging. Arthritis Rheum. 1999.

25. Bossuyt PM, Reitsma JB, Bruns DE, Gatsonis CA, Paul P, Irwig L, et al. STARD 2015: an updated list of essential items for. BMJ Open. 2015;351(h5527):1-9.

26. Altman R, Alarcon G, Appelrouth D, Bloch D, Borenstein D, Brandt K, et al. The American College of Rheumatology criteria for the classification and reporting of osteoarthritis of the hand. Arthritis Rheum. 1990.

27. Bruce B, Fries JF. The Health Assessment Questionnaire (HAQ). Clin Exp Rheumatol. 2005;23(5 SUPPL. 39).

28. Maksymowych W, Mallon CMS, et al. Development and validation of the Spondyloarthritis Research Consortium of Canada (SPARCC) Enthesitis Index. Ann Rheum Dis. 2009;68(6):948-53.

29. Terslev L, Naredo E, Aegerter P, Wakefield RJ, Backhaus M, Balint P, et al. Scoring ultrasound synovitis in rheumatoid arthritis: A EULAR-OMERACT ultrasound taskforce-Part 2: Reliability and application to multiple joints of a standardised consensus-based scoring system. RMD Open. 2017;3(1).

30. Ellegaard K, Torp-Pedersen S, Lund H, Henriksen M, Terslev L, Jensen PS, et al. Quantification of colour Doppler activity in the wrist in patients with rheumatoid arthritis - The reliability of different methods for image selection and evaluation. Ultraschall der Medizin. 2008;29(4):393-298.

31. Shrout PE, Fleiss JL. Intraclass correlations: Uses in assessing rater reliability. Psychol Bull. 1979;86(2):420-8.

32. Cicchetti DV, Guidelines. Criteria, and Rules of Thumb for Evaluating Normed and Standardized Assessment Instruments in Psychology. Psychol Assess. 1994;6(4):284-90.

33. Aydin SZ, Castillo-Gallego C, Ash ZR, Marzo-Ortega H, Emery P, Wakefield RJ, et al. Ultrasonographic assessment of nail in psoriatic disease shows a link between onychopathy and distal interphalangeal joint extensor tendon enthesopathy. Dermatology. 2013;225(3):231-5.

34. McGonagle D, Hermann KGA, Tan AL. Differentiation between osteoarthritis and psoriatic arthritis: Implications for pathogenesis and treatment in the biologic therapy era. Rheumatology (United Kingdom). 2015.

35. Scanzello CR. Role of low-grade inflammation in osteoarthritis. Current Opinion in Rheumatology. 2017.

36. Penatti A, Facciotti F, De Matteis R, Larghi P, Paroni M, Murgo A, et al. Differences in serum and synovial CD $4+T$ cells and cytokine profiles to stratify patients with inflammatory osteoarthritis and rheumatoid arthritis. Arthritis Res Ther. 2017.

37. Terslev L, Naredo E, lagnocco A, Balint PV, Wakefield RJ, Aegerter P, et al. Defining enthesitis in spondyloarthritis by ultrasound: Results of a delphi process and of a reliability reading exercise. Arthritis Care Res. 2014;66(5).

38. Schraml C, Schwenzer NF, Martirosian P, Koetter I, Henes JC, Geiger K, et al. Assessment of Synovitis in Erosive Osteoarthritis of the Hand using DCE-MRI and Comparison with that in its Major Mimic, the 
Psoriatic Arthritis. Acad Radiol. 2011.

39. Schwenzer NF, Kötter I, Henes JC, Schraml C, Fritz J, Claussen CD, et al. The role of dynamic contrastenhanced MRI in the differential diagnosis of psoriatic and rheumatoid arthritis. Am J Roentgenol. 2010.

40. Boesen M, Kubassova O, Sudoł-Szopińska I, Maas M, Hansen P, Nybing JD, et al. MR Imaging of Joint Infection and Inflammation with Emphasis on Dynamic Contrast-Enhanced MR Imaging. PET Clinics. 2018.

41. Zhang Y, Niu J, Kelly-Hayes M, Chaisson CE, Aliabadi P, Felson DT. Prevalence of symptomatic hand osteoarthritis and its impact on functional status among the elderly: The framingham study. Am J Epidemiol. 2002.

42. Haugen IK, Englund M, Aliabadi P, Niu J, Clancy M, Kvien TK, et al. Prevalence, incidence and progression of hand osteoarthritis in the general population: The Framingham Osteoarthritis Study. Ann Rheum Dis. 2011.

\section{Figures}

\begin{tabular}{|c|c|c|}
\hline $\begin{array}{l}\text { Assessed for eligibility PsO } \\
\qquad(n=14)\end{array}$ & $\begin{array}{l}\text { Assessed for eligibility PsA } \\
\qquad(n=70)\end{array}$ & $\begin{array}{l}\text { Assessed for eligibility } O A \\
(n=14)\end{array}$ \\
\hline \multicolumn{2}{|c|}{$\begin{array}{l}\text { Excluded ( } \mathbf{n}=\mathbf{2 3} \text { ) } \\
\text {-Not meeting inclusion criteria (PSA n=6, PsO n=1). } \\
\text {-Practicalfeasibilityjob related hindrances (PSA } n=7 \text {, PSO } n=1, \text { OA n=1) } \\
\text { - Other reasons/no response (PSA } n=7 \text { ) }\end{array}$} & \\
\hline Included $\mathrm{PsO}_{\mathrm{s}}=12$ & Included PsA n=50 & Included OA n=13 \\
\hline 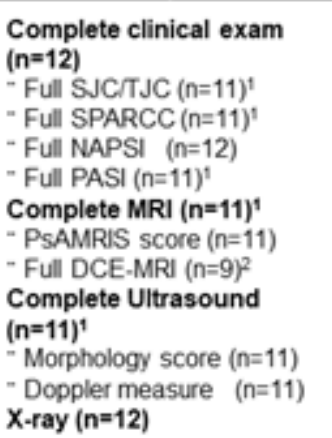 & $\begin{array}{l}\text { Complete clinical exam } \\
\text { (n=50) } \\
\text { - SJC/TJC }(n=50) \\
\text { - SPARCC }(n=50) \\
\text { - NAPSI }(n=50) \\
\text { - PASI }(n=50) \\
\text { Complete MRI }(n=44)^{2} \\
\text { - PSAMRIS SCore }(n=42)^{3} \\
\text { - Full DCE-MRI }(n=42)^{3} \\
\text { Complete Ultrasound } \\
\text { (n=50) } \\
- \text { Morphology score }(n=50) \\
\text { - Doppler measure } \quad(n=47)^{4} \\
\text { X-ray }(n=49)^{5}\end{array}$ & $\begin{array}{l}\begin{array}{l}\text { Complete clinical exam } \\
(n=13)\end{array} \\
\text {-Full SJCTIJC }(n=13) \\
\text { - Full SPARCC }(n=13) \\
\text { - Full NAPSI }(n=13) \\
\text { - Full PASI }(n=13) \\
\text { Complete MRI }(n=12)^{2} \\
\text { - PsAMRIS SCore }(n=12) \\
\text { - Full DCE-MRI }(n=12) \\
\text { Complete Ultrasound } \\
(n=13) \\
\text { - Morphology score }(n=13) \\
\text { - Doppler measure }(n=13) \\
\text { X-ray }(n=13)\end{array}$ \\
\hline
\end{tabular}

\section{Figure 1}

Flowchart of study participants. 1 Reasons for declining further participation: No response after first examination day (PsA $n=1, P s O=1) .2$ No MRI: Obesity/ claustrophobic (PsA $n=3, O A=1)$, MRI without 
Gadolinium contrast (PsA n=3, PsO n=2). 3 Finger out of scanning plane (PsA n=2). 4 Image not acquired: due to deformity (PsA $n=1)$, Missing per US-protocol (PsA $n=1)$. No Dopplerclip: Poor quality (PsA $n=1) .5$ Image of non-target hand $(n=1)$. Abbreviations: PsA: psoriatic arthritis, PsO: skin psoriasis, OA: osteoarthritis, MRI: Magnetic Resonance Imaging. SJC/TJC: swollen joint count/tender joint count. Flow chart template modified from http://www.equator-network.org/reporting-guidelines/stard/

DIP Joint US Synovitis score

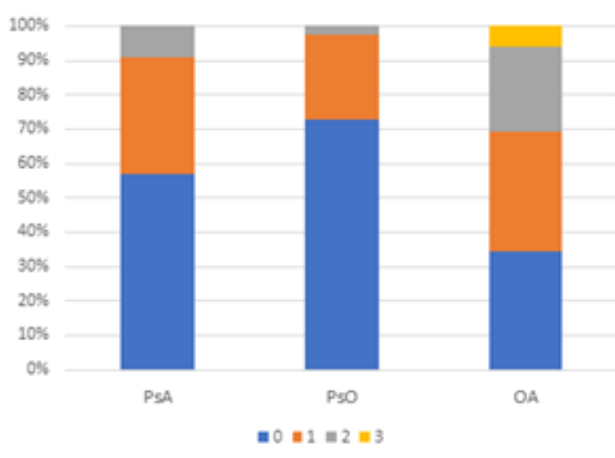

DIP Joint US Erosion Score

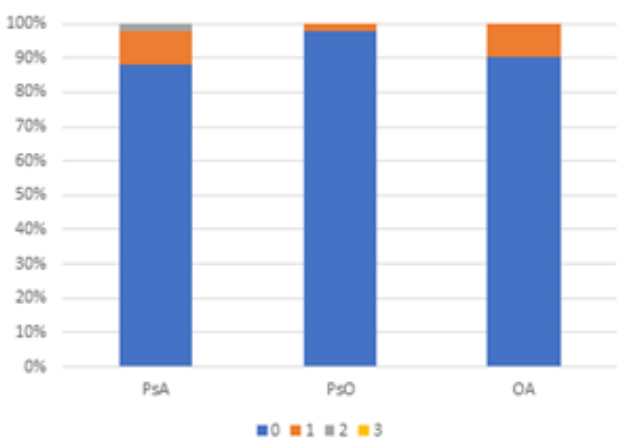

DIP Joint US Doppler score

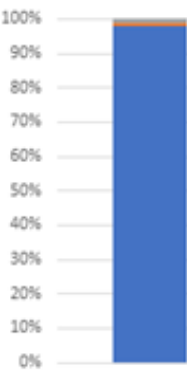

PsA

Dip Joint US New Bone Formation Score

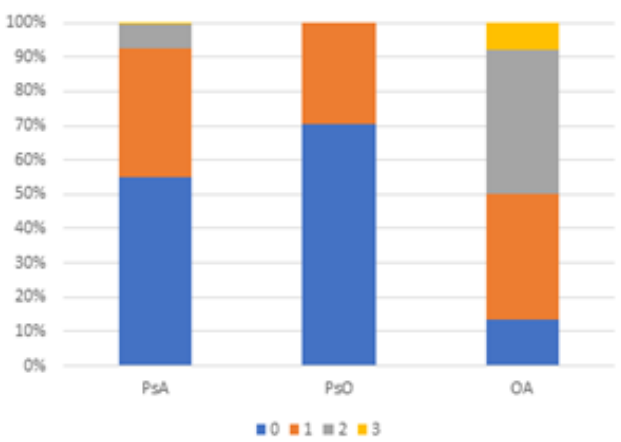

US Extensor Tendon Enthesitis
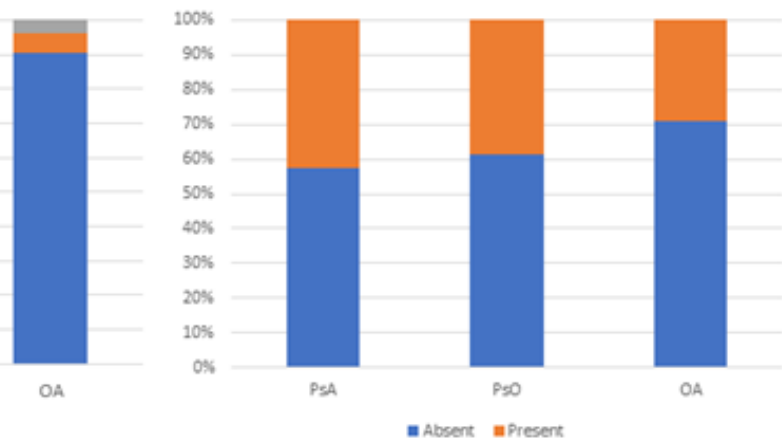

DIP Joint US Peritendonitis

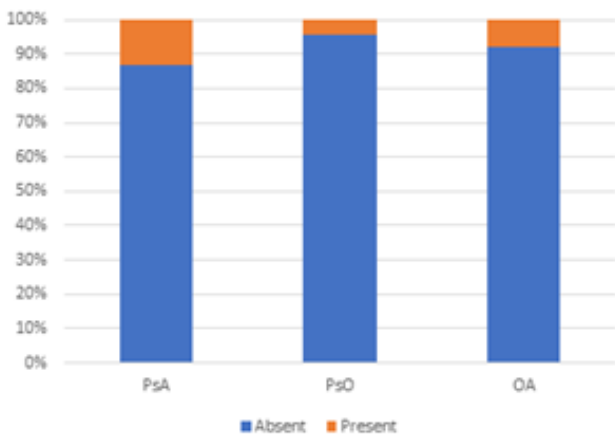

Figure 2

Percentual distributions of ultrasound findings of the distal interphalangeal joints among patients with PsA, PsO and hand OA according to the grade of involvement 0-3 or as absent or present. Abbreviations: DIP: Distal Interphalangeal, US: ultrasound, PsA: psoriatic arthritis, PsO: skin psoriasis, OA: osteoarthritis. 


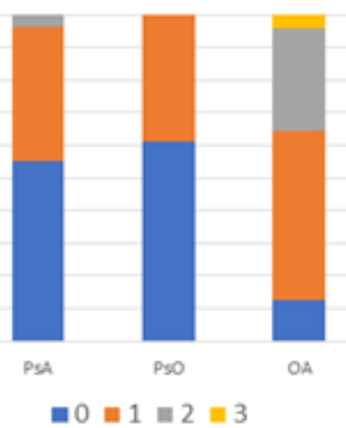

MRI Tenosynovitis score

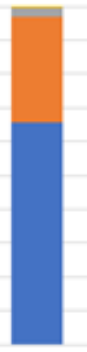

PsA

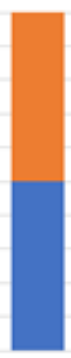

P9o

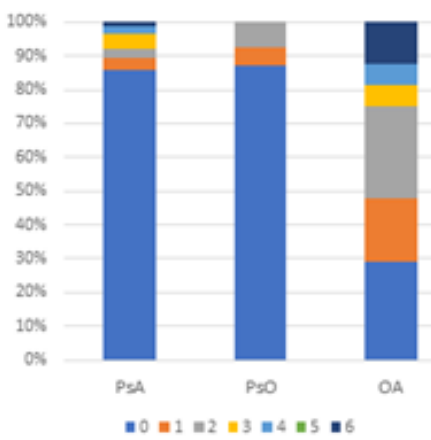

MRI total DIP-Joint periarticular inflammation
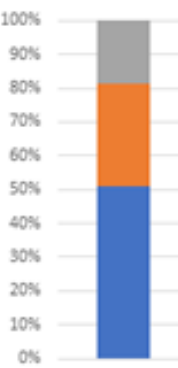

PSA

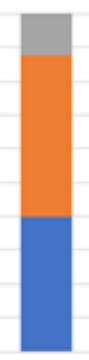

Pso

$m=1=2$

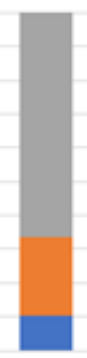

$O A$

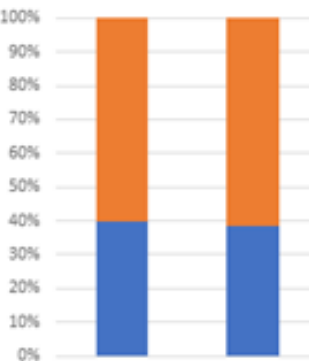

Psa

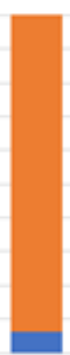

MRI total DIP-Joint Erosions

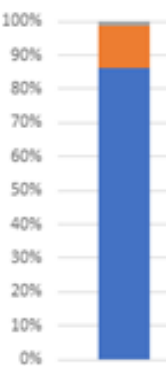

PSA

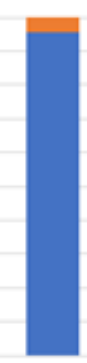

$P 90$

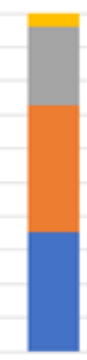

OA

$\| 0=1=2=3$

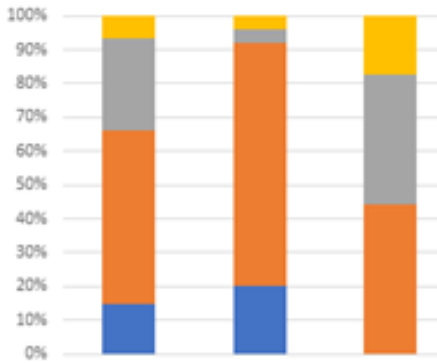

$P=0$

OA

X-ray DIP-joint erosions
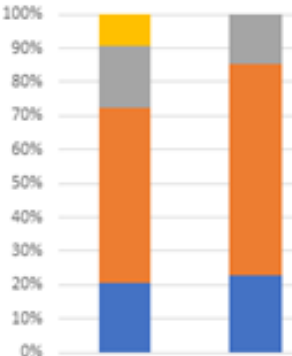

PsO

- $0=1=2=3$

\section{Figure 3}

Percentual distributions of magnetic resonance imaging and X-ray findings of the distal interphalangeal joint among patients with PsA, PsO and hand OA according to the grade of involvement or as absent or present. Abbreviations: DIP: Distal Interphalangeal, MRI: magnetic resonance imaging, PsA: psoriatic arthritis, PsO: skin psoriasis, OA: osteoarthritis.

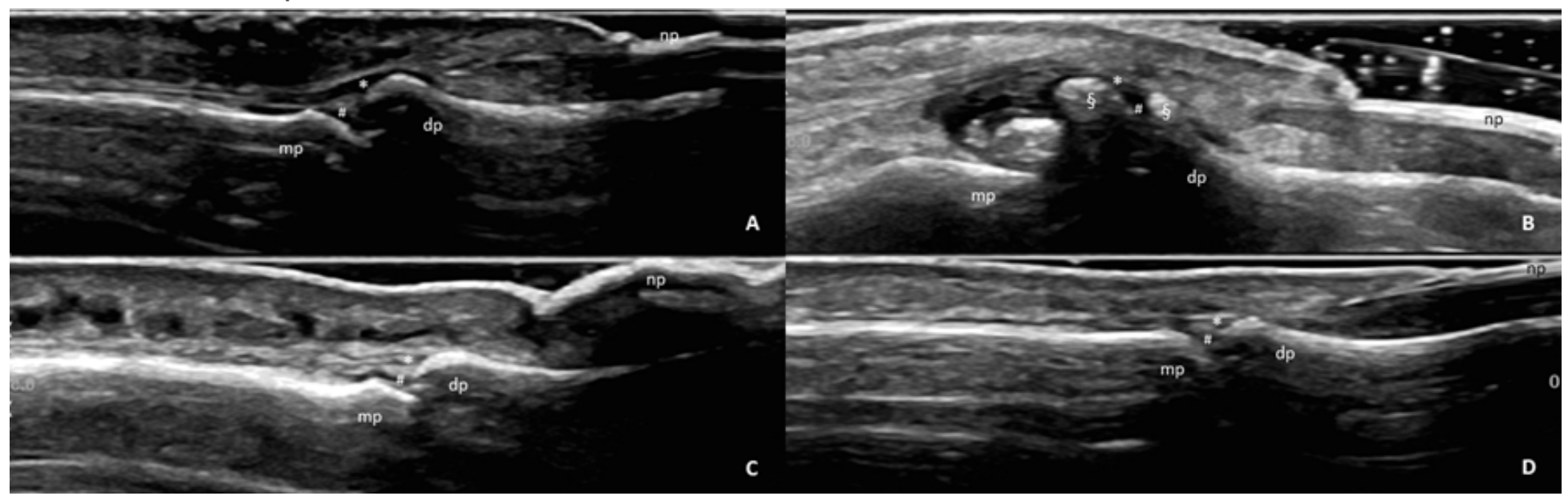

Figure 4

Ultrasonographic image of the synovio-entheseal-complex. Dorsal longitudinal scan. A: Patients with psoriatic arthritis with loss of normal fibrillary architecture at extensor tendon insertion $\left({ }^{*}\right)$ at distal phalanx (dp) and effusion at the distal interphalangeal joint (\#). B: Patient with osteoarthritis with severe 
new bone formation (§), effusion and synovial hypertrophy of the distal interphalangeal joint (\#). C:

Patients with skin psoriasis with severe thickening and loss of trilaminar structure of the nail plate (np). D: Healthy control demonstrating normal fibrillar architecture of the exensor tendon $\left(^{\star}\right)$ and a healthy nail plate $(\mathrm{np})$ with trilaminar structure as two hyperechoic lines surrounding an anechoic line. $\mathrm{mp}$ : medial phalanx.

\section{Supplementary Files}

This is a list of supplementary files associated with this preprint. Click to download.

- SupplementaryDataS1ProtocolMIDASver5.2.pdf

- SupplementaryTableS2eligibilitycriteria.docx

- SupplementaryDataS3Probecomparison.pdf

- SupplementaryDataS4ultrasoundprotocol.docx

- SupplementaryDataS5MRIprotocolincludingDCEMRI.docx

- SupplementaryDataS6Xrayprotocol.docx

- SupplementaryTableS7ICCandKappatables.docx

- SupplementaryTableS8AUCPsADiagnosis.docx 